\title{
Chemistry under nanoconfinement
}

\author{
When the size of the vessel controls the outcome of a reaction.
}

( ne of the tenets of nanoscience is the study of systems whose properties change with size. The image of several vials lined up with quantum dot solutions glowing from red to blue is emblematic. Less familiar is the case for chemical reactions. If you carry out your reaction in a $500 \mu \mathrm{l}$ or in a $10 \mathrm{ml}$ flask the outcome is going to be the same. But if you start decreasing the size of the vessel to a point in which the interaction between the walls of the container and your molecules becomes relevant, then the size of the container will play a role in your chemistry. In this issue, we publish a Review Article highlighting various size effects in chemistry.

Chemistry under a nanoconfined space is not a conceptually new proposition. Zeolites, for example, are used routinely to catalyse and control the outcome of oil cracking reactions in industry. In academia, studies of reactions within micelles and macromolecules date back to the 1970s. In biology, nanoconfinement is the key reason for the selectivity of enzymatic reactions. Recently, however, the field has expanded significantly, showing an ever-increasing level of sophistication. The size and shape of zeolites can improve the stereoselectivity and the yield of bioplastics production by preventing racemization and the formation of side products. Containers can be engineered to pre-organize reagents next to a catalytic site for enhanced reaction rates. Containers can even be made to assemble and disassemble by means of a physicochemical signal; in this way, the formation of a certain desired product can be triggered at a specific moment in time. Nanocontainers are also useful to isolate and study reactive intermediates with immediate benefits in the design of more efficient catalysts; such is the case for catalysts whose activity is similar to that of natural enzymes (so-called, nanozymes) that aim to marry the kinetics of natural enzymes with greater substrate versatility.

Another important reason to study chemical reactions under nanoconfined spaces is the direct role compartmentalization of biochemical reactions plays in the machinery of life. If we wish to understand life, out-ofequilibrium systems and make materials with life-like behaviour, chemistry under nanoconfinement remains a frontier to be conquered.

Published online: 17 April 2020 https://doi.org/10.1038/s41565-020-0664-y

\section{The race against COVID-19}

\author{
The identification and isolation of individuals with COVID-19 can help to flatten the epidemic curve and win us time \\ to wait for the vaccine development and production, and antiviral drug therapies.
}

T he COVID-19 pandemic concerns people all over the world. It has forced closedown of borders, lockdown of cities, proactive closure of schools/ universities/labs in multiple countries, cancellation of events including scientific conferences and triggered a series of chain reactions, harming the global economy.

Various biopharmaceutical companies and organizations, such as Moderna/ National Institutes of Health (NIH), Medicago, Inovio, Clover/GSK and the Chinese Academy of Military Medical Sciences have announced potential COVID-19 vaccine candidates. More excitingly, Moderna/NIH and the Chinese Academy of Military Medical Sciences have begun their clinical trials. However, health officials estimate that it may still take 1 year to 18 months to achieve the production and distribution of vaccines ${ }^{1,2}$. Repurposing existing antiviral strategies and vaccines could provide a shortcut. Writing in Nature Nanotechnology, Hu et al. comment on the fact that chloroquine, which is a US Food and Drug Administrationapproved drug for the treatment of malaria, inhibits endocytosis of nanoparticles in the same size range as viruses and discuss the potential of this drug against COVID- $19^{3}$. Indeed, chloroquine has already been put in practice for the treatment of COVID-19 patients in China since 19 February 2020, according to the sixth version of Guideline Over the Diagnosis and Treatment of COVID-194. The guideline was updated on 3 March, still including chloroquine. Chloroquine is not highly efficient but it is beneficial and side effects are minor, according to Chinese health officials ${ }^{5,6}$. The clinical trials in China also suggests favipiravir, the antiviral drug developed by Toyama Chemical to treat flu, shows good clinical efficacy against COVID-19 and is being included into the treatment plan $^{7}$. Doctors in Japan now are also using favipiravir to treat COVID-19 patients with mild to moderate symptoms to inhibit the multiplication of the virus ${ }^{8}$. But the drug does not work that well on people with more severe symptoms, noted a Japanese health ministry source ${ }^{8}$.

Investigations and tests of several potential drugs and therapies are also being conducted. Remdesivir, developed by Gilead Sciences, showed promising in vitro and in vivo performance against the coronaviruses that caused Middle East respiratory syndrome (MERS) in 2012 and the coronavirus that caused severe acute respiratory syndrome (SARS) in 2003, and has been described as the "most promising candidate" against COVID-199-11. Results of clinical trials of remdesivir, currently under way in China ${ }^{12}$, are expected to be released at the end of April, which hopefully will be followed by the results of clinical trials conducted in the US and other countries ${ }^{13,14}$. Moreover, early clinical trials in China suggest that convalescent plasma therapy, which has previously been used to treat SARS and Ebola infections ${ }^{15}$, is effective in 
curing high-risk patients with COVID- $19^{16}$. Takeda has also begun with the development of a plasma-derived therapy. In addition, Eli Lilly/AbCellera, Biogen/Vir and Regeneron have started to work on antibody-based therapies.

However, the development of prophylactics and therapeutics requires time to ensure adequate preclinical and clinical testing and ultimately, efficacy against COVID-19. The spread of COVID-19 needs to be slowed down to win us time to wait for that. Otherwise, the rapidly accumulated number of patients is going to overwhelm the healthcare capacity, resulting in an increased fatality rate. Various countries have recognized the most effective route to mitigation to be identification and isolation of infected people as well as tracing of their close contacts. For example, South Korea substantially scaled-up the number of COVID-19 tests and isolations and could, thereby, decrease the spreading of the virus after the large outbreak. The South Korean government has quickly given approval to four companies for the manufacturing of test kits following the initial COVID-19 outbreak reported in Wuhan, China, and within weeks has set up a network capable of running 20,000 tests per day ${ }^{17}$. They further built drive-through centres, at which swab tests can be collected for COVID-19 diagnosis. Indeed, the car serves as an isolated waiting room. This model is now being followed by the US, which has also greatly improved testing capacities.

Immediate and accurate testing was a big challenge at the beginning of the outbreak in Wuhan, Hubei, the epicentre of COVID19 in China. Nucleic acid test kits were not available in Hubei province until January 16. The initial unavailability of test kits and frequent false negatives at the beginning of the outbreak resulted in more infections than the official figures indicated and delayed the isolation of infected patients. On 12 February 2020, Chinese health authorities modified the guidelines to include computerized tomography scans for the diagnosis and confirmation of infections, which led to an update of 14,840 new cases in a single day on 13 February, a daily record, with a new category of patients 'confirmed by clinical diagnosis ${ }^{18}$. However, this category was then removed on the following day, as the guideline was again updated stating that a positive nucleic test result remains the only way to confirm infection, because pulmonary symptoms develop gradually and may be caused by other infections. Fortunately, adequate test kit supply could be guaranteed soon afterwards, as the Chinese National Medical Products Administration has fasttracked the approval of 10 new coronavirus nucleic acid detection reagents and 6 antibody detection reagents (http://www. nmpa.gov.cn/WS04/CL2042/).

Antibody tests need to be used in conjunction with other testing regimes to confirm infections, because antibody titres remain below the detection limit during the first few days of infection. However, straightforward operation of chemiluminescence immunoassays and colloidal gold nanoparticle probe-based immunoassays enable fast identification of asymptomatic patients and patients with mild symptoms, which allows the subsequent contact tracing/mandated quarantines without delay. Scientists are currently also developing antibody test kits to allow self-diagnosis at home ${ }^{19}$. The nucleic acid test based on a reverse transcription polymerase chain reaction and next-generation sequencing in combination with antibody detection can greatly improve the identification and isolation rate. Moreover, quantification of antibodies against the new coronavirus can also be used to assess whether a convalescent survivor is qualified to donate convalescent plasma. In addition, diagnosis based on the identification of viral proteins, is also on its way, which will allow fast diagnosis without any delay using a simple set-up. The availability of more fast, accurate and portable tests will further improve the identification and isolation.

It is also worth noting that, aside from the identification and isolation, how individuals respond to the advice of social distancing and mandatory quarantine, is equally important ${ }^{20}$. The race against COVID-19 will therefore be won only if science is supported by the necessary social behaviour.

Published online: 17 April 2020

https://doi.org/10.1038/s41565-020-0680-y
References

1. Lovelace, B. First human trial for coronavirus vaccine begins Monday in the US. CNBC https://www.cnbc.com/2020/03/16 first-human-trial-for-coronavirus-vaccine-begins-monday-inthe-us.html (2020).

2. Lovelace, B. \& Higgins-Dunn, N. Human trials for a coronavirus vaccine could begin 'within a few weeks', top US health official says. CNBC https://www.cnbc.com/2020/03/12/human-trialstesting-coronavirus-vaccine-could-begin-within-a-few-weeks. html (2020).

3. Hu. T. Y., Frieman, M. \& Wolfram, J. Nat. Nanotechnol. https://doi.org/10.1038/s41565-020-0674-9 (2020).

4. Guideline Over the Diagnosis and Treatment of COVID-19 version 6 (in Chinese) (The State Council of the People's Republic of China, 2020); http://www.gov.cn/zhengce/zhengceku/2020-02/19/ content_5480948.htm

5. Chloroquine phosphate is beneficial in the treatment of new coronary pneumonia (in Chinese). Sciencenet http://news sciencenet.cn/htmlnews/2020/2/435861.shtm (2020).

6. New use of old medicine: Past and present of chloroquine phosphate (in Chinese). CCTV http://big5.cctv.com/gate/big5/ news.cctv.com/2020/02/28/ARTI7bHiISLdC6JMBiCgOxIs200228. $\operatorname{shtml}(2020)$.

7. Favipiravir shows good clinical efficacy in treating COVID-19: official. XINHUANET http://www.xinhuanet.com/ english/2020-03/17/c_138888102.htm (2020)

8. McCurry, J. Japanese flu drug 'clearly effective' in treating coronavirus, says China. The Guardian (18 March 2020); https://www.theguardian.com/world/2020/mar/18/japanese-fludrug-clearly-effective-in-treating-coronavirus-says-china

9. Harrison, C. Nat. Biotechnol. https://doi.org/10.1038/d41587-02000003-1 (2020).

10. Devlin, H. \& Sample, I. Hopes rise over experimental drug's effectiveness against coronavirus. The Guardian (10 March 2020); https://www.theguardian.com/world/2020/mar/10/hopes-riseover-experimental-drugs-effectiveness-against-coronavirus

11. Knapp, A. Coronavirus drug update: The latest info on pharmaceutical treatments and vaccines. Forbes https://www. forbes.com/sites/alexknapp/2020/03/13/coronavirus-drugupdate-the-latest-info-on-pharmaceutical-treatments-andvaccines/\#205added21db (2020).

12. Mild/moderate 2019-nCoV remdesivir RCT ClinicalTrials.gov http://apps.who.int/trialsearch/Trial2. aspx? TrialiD=NCT04252664 (2020).

13. Study to evaluate the safety and antiviral activity of remdesivir (GS-5734*) in participants with severe coronavirus disease (COVID-19). ClinicalTrials.gov https://clinicaltrials.gov/ct2/ show/record/NCT04292899 (2020).

14. Adaptive COVID-19 Treatment Trial (ACTT). ClinicalTrials. gov https://clinicaltrials.gov/ct2/show/NCT04280705 (2020).

15. Use of Convalescent Whole Blood or Plasma Collected from Patients Recovered from Ebola Virus Disease for Transfusion, as an Empirical Treatment During Outbreaks: Interim Guidance for National Health Authorities and Blood Transfusion Services WHO/HIS/SDS/2014.8 (World Health Organization, 2014).

16. China puts 245 COVID-19 patients on convalescent plasma therapy. XINHUANET http://www.xinhuanet.com/ english/2020-02/28/c_138828177.htm (2020).

17. Bicker, L. Coronavirus in South Korea: How 'trace, test and treat' may be saving lives. $B B C$ https://www.bbc.com/news/worldasia-51836898 (2020)

18. Patton D. \& Stanway, D. China’s Hubei province sees surge in coronavirus deaths on switch to new methodology. Reuters https://www.reuters.com/article/us-china-health-hubei-toll/ chinas-hubei-province-sees-surge-in-coronavirus-deaths-onswitch-to-new-methodology-idUSKBN207010 (2020).

19. Park, A. The first U.S. company has announced an upcoming home COVID-19 test. TIME https://time.com/5805953/homecovid-19-test-everlywell/ (2020).

20. Anderson, R. M., Heesterbeek, H., Klinkenberg, D. \& Déidre Hollingsworth, T. Lancet 395, 931-934 (2020). 